\title{
Existence, Uniqueness, and Stability of Solutions of Systems of Complex Integrodifferential Equations on Complex Planes
}

\author{
HEWA SELMAN FARIS ${ }^{1}$ \\ Department of Mathematics \\ Duhok University \\ Duhok city \\ IRAQ \\ RAAD NOORI BUTRIS ${ }^{2}$ \\ Department of Mathematics \\ Duhok University \\ Duhok city \\ IRAQ
}

\begin{abstract}
In this paper, we investigate the existence, uniqueness, and stability of solutions for a class of systems of non-linear complex Integrodifferential equations on complex planes. Based on the complex Integrodifferential equations, the iterative sequences for approximating the solutions are derived and several theorems about the existence and the forms of entire solutions are established. Finally, numerical results are illustrated from an example to confirm the veracity and applicability of the main problem whose exact solutions are available.
\end{abstract}

Key-Words: - Complex Integrodifferential system, existence, uniqueness, stability, analytic \& holomorphic functions, complex plane.

Received: May 15, 2021. Revised: January 17, 2022. Accepted: February 12, 2022. Published: March 3, 2022.

\section{Introduction}

Complex differential equations (CDEs) are increasingly prevalent in physics, engineering, electrics, and other fields that are devoted by many authors such as A L. Cauch, B. Riemann, and K. Weierstrass [10]. The concept of system of complex-differentiability will be introduced exactly as was that of differentiability on, $\mathbb{R}$ [12]. The complex-valued functions which are complexdifferentiable throughout a domain in $\mathbb{C}$ are called analytic (holomorphic) and function theory is usually understood to be the study of such functions [5].

Many works in complex differential equations, complex integral equations, and complex Integrodifferential equations have been demonstrated by authors. The aim of this study is to investigate and develop complex Integrodifferential equations. This paper has been organized as: The first section is intended to provide a presentation of basic definitions and properties of complex concepts suitable for the basis employed in many branches of mathematics and physics [1], [2] and [7].

In Sections 2, 3, and 4, consequently, the existence, uniqueness, and stability of solutions of system (1) have been obtained [4], [6] and [14]. The last section contains an example for the validity of the solutions of (CIDF) of the system (1) and we picture them geometrically, as curves for more clarity in the complex planes [8] and [9].

Suppose that the non-linear complex Integrodifferential equations are defined within a system as: 


$$
\left.\begin{array}{c}
\frac{d w\left(z ; w_{0}, \omega_{0}\right)}{d z}=\left(A_{1}+B_{1}(z)\right) w\left(z ; w_{0}, \omega_{0}\right) \\
+\left(A_{2}+B_{2}(z)\right) \omega\left(z ; w_{0}, \omega_{0}\right) \\
+f\left(z ; w\left(z ; w_{0}, \omega_{0}\right), \omega\left(z ; w_{0}, \omega_{0}\right)\right) \\
\frac{d \omega\left(z ; w_{0}, \omega_{0}\right)}{d z}=\left(C_{1}+D_{1}(z)\right) w\left(z ; w_{0}, \omega_{0}\right) \\
+\left(C_{2}+D_{2}(z)\right) \omega\left(z ; w_{0}, \omega_{0}\right) \\
+g\left(z ; w\left(z ; w_{0}, \omega_{0}\right), \omega\left(z ; w_{0}, \omega_{0}\right)\right)
\end{array}\right\}
$$

with, initial conditions, $w\left(0, w_{0}, \omega_{0}\right)=w_{0}$ and $\omega\left(0, w_{0}, \omega_{0}\right)=\omega_{0} \quad$ consequently, where, $w_{0} \in$ $\mathbb{C}^{n}, \omega_{0} \in \mathbb{C}^{m}, \quad w\left(z ; w_{0}, \omega_{0}\right) \in \Omega_{0} \quad$ and $\omega\left(z ; w_{0}, \omega_{0}\right) \in \Omega_{1}$. The compact subsets, $\Omega_{0}$ and $\Omega_{1}$ are complex Euclidean spaces $\mathbb{C}^{n}$ and, $\mathbb{C}^{m}[3]$.

Let $\quad f\left(z ; w\left(z ; w_{0}, \omega_{0}\right), \omega\left(z ; w_{0}, \omega_{0}\right)\right) \quad$ and $g\left(z ; w\left(z ; w_{0}, \omega_{0}\right), \omega\left(z ; w_{0}, \omega_{0}\right)\right)$ be holomorphic complex-valued vector functions that defined on domain

$\Omega=(z ; w, \omega) \in \mathbb{C} \times \mathbb{C}^{n} \times \mathbb{C}^{m}$

Where, $\left\|z-z_{0}\right\| \leq a,\left\|w\left(z ; w_{0}, \omega_{0}\right)-w_{0}\right\| \leq$ $\beta_{w}$ and $\left\|\omega\left(z ; w_{0}, \omega_{0}\right)-\omega_{0}\right\| \leq \beta_{\omega}$.

We consider the solutions $w\left(z ; w_{0}, \omega_{0}\right)$ and, $\omega\left(z ; w_{0}, \omega_{0}\right)$ exactly from problem $(1)$ as:

$w\left(z ; w_{0}, \omega_{0}\right)=w_{0} e^{A_{1} z}+\int_{0}^{z} e^{A_{1}(z-s)} \zeta(s) d s$,

where, $\quad \zeta(z)=B_{1}(z) w\left(z ; w_{0}, \omega_{0}\right)+\left(A_{2}+\right.$ $\left.B_{2}(z)\right) \omega\left(z ; w_{0}, \omega_{0}\right)+$ $f\left(z ; w\left(z ; w_{0}, \omega_{0}\right), \omega\left(z ; w_{0}, \omega_{0}\right)\right)$.

$\omega\left(z ; w_{0}, \omega_{0}\right)=\omega_{0} e^{C_{2} z}+\int_{0}^{z} e^{C_{2}(z-s)} \xi(s) d s$,

where, $\quad \xi(z)=\left(C_{1}+D_{1}(z)\right) w\left(z ; w_{0}, \omega_{0}\right)+$ $D_{2}(z) \omega\left(z ; w_{0}, \omega_{0}\right)+$

$g\left(z ; w\left(z ; w_{0}, \omega_{0}\right), \omega\left(z ; w_{0}, \omega_{0}\right)\right)$.

The holomorphic vector functions, $f\left(z ; w\left(z ; w_{0}, \omega_{0}\right), \omega\left(z ; w_{0}, \omega_{0}\right)\right), \quad$ and $g\left(z ; w\left(z ; w_{0}, \omega_{0}\right), \omega\left(z ; w_{0}, \omega_{0}\right)\right)$ require the following conditions and inequalities:

$\left.\left\|w_{0}\right\|\left\|e^{A_{1} z}-I\right\|=Q_{1}(z)\right\}$

$\left.\left\|\omega_{0}\right\|\left\|e^{C_{2} z}-I\right\|=Q_{2}(z)\right\}$

$\|\zeta(z)\| \leq \vartheta_{1}(z)$

$\left.\|\xi(z)\| \leq \vartheta_{2}(z)\right\}$

$$
\begin{gathered}
\| f\left(z ; w_{2}\left(z ; w_{0}, \omega_{0}\right), \omega_{2}\left(z ; w_{0}, \omega_{0}\right)\right)- \\
f\left(z ; w_{1}\left(z ; w_{0}, \omega_{0}\right), \omega_{1}\left(z ; w_{0}, \omega_{0}\right)\right) \| \leq \\
\Gamma_{1}\left\|w_{2}\left(z ; w_{0}, \omega_{0}\right)-w_{1}\left(z ; w_{0}, \omega_{0}\right)\right\|+ \\
\Gamma_{2}\left\|\omega_{2}\left(z ; w_{0}, \omega_{0}\right)-\omega_{1}\left(z ; w_{0}, \omega_{0}\right)\right\| \\
\| g\left(z ; w_{2}\left(z ; w_{0}, \omega_{0}\right), \omega_{2}\left(z ; w_{0}, \omega_{0}\right)\right)- \\
g\left(z ; w_{1}\left(z ; w_{0}, \omega_{0}\right), \omega_{1}\left(z ; w_{0}, \omega_{0}\right)\right) \| \leq \\
\quad \Sigma_{1}\left\|w_{2}\left(z ; w_{0}, \omega_{0}\right)-w_{1}\left(z ; w_{0}, \omega_{0}\right)\right\|+ \\
\Sigma_{2}\left\|\omega_{2}\left(z ; w_{0}, \omega_{0}\right)-\omega_{1}\left(z ; w_{0}, \omega_{0}\right)\right\|
\end{gathered}
$$

$\forall$, solutions $w, w_{1}, w_{2} \in \Omega_{0}$ and, $\omega, \omega_{1}, \omega_{2} \in \Omega_{1}$, where $Q_{1}, Q_{2}, \vartheta_{1}, \vartheta_{2}, \Gamma_{1}, \Gamma_{2}, \Sigma_{1}$ and $\Sigma_{2}$ are positive constants.

The complex-valued matrices $A_{1}=\left(A_{1: j, k}\right)$, $A_{2}=\left(A_{2: j, k}\right), \quad B_{1}=\left(B_{1: j, k}\right), \quad B_{2}=\left(B_{2: j, k}\right)$, $C_{1}=\left(C_{1: j, k}\right), \quad C_{2}=\left(C_{2: j, k}\right), \quad D_{1}=\left(D_{1: j, k}\right) \quad$ and $D_{2}=\left(D_{2: j, k}\right)$ are positive, for all $j, k=1,2, \ldots, n$ and $\|w\|=\max _{z \in[0, Z]}\left|w_{j}\right|$.

From the complex Euclidean spaces $\mathbb{C}^{n}$ and, $\mathbb{C}^{m}$ non-empty sets are defined as

$$
\begin{aligned}
& \Omega_{f}=\Omega_{0}-\beta_{w}=\Omega_{0}-\left(Q_{1}(z)+z R_{1} \vartheta_{1}(z)\right) \\
& \left.\Omega_{g}=\Omega_{1}-\beta_{\omega}=\Omega_{1}-\left(Q_{2}(z)+z R_{2} \vartheta_{2}(z)\right)\right\} \\
& \left.\begin{array}{l}
\left\|e^{A_{1}(z-s)}\right\|=\mathrm{R}_{1} \\
\left\|e^{C_{2}(z-s)}\right\|=R_{2}
\end{array}\right\}
\end{aligned}
$$

We defined the complex sequences $\left\{w_{m}\left(z ; w_{0}, \omega_{0}\right)\right\}_{m=0}^{\infty}$ and, $\left\{\omega_{m}\left(z ; w_{0}, \omega_{0}\right)\right\}_{m=0}^{\infty}$ as:

$$
w_{m+1}\left(z ; w_{0}, \omega_{0}\right)=w_{0} e^{A_{1} z}+\int_{0}^{z} e^{A_{1}(z-s)} \zeta_{m}(s) d s
$$

While, $\quad w\left(0, w_{0}, \omega_{0}\right)=w_{0}$ and, $m=0,1,2, \ldots$, where $\quad \zeta_{m}(z)=B_{1}(z) w_{m}\left(z ; w_{0}, \omega_{0}\right)+$ $\left(A_{2}+B_{2}(z)\right) \omega_{m}\left(z ; w_{0}, \omega_{0}\right)+$ $f\left(z ; w_{m}\left(z ; w_{0}, \omega_{0}\right), \omega_{m}\left(z ; w_{0}, \omega_{0}\right)\right)$.

$\omega_{m+1}\left(z ; w_{0}, \omega_{0}\right)=\omega_{0} e^{C_{2} z}+\int_{0}^{z} e^{C_{2}(z-s)} \xi_{m}(s) d s$

While, $\quad \omega\left(0, w_{0}, \omega_{0}\right)=\omega_{0} \quad$ and, $\quad m=0,1,2, \ldots$, where $\quad \xi_{m}(z)=\left(C_{1}+D_{1}(z)\right) w_{m}\left(z ; w_{0}, \omega_{0}\right)+$ $D_{2}(z) \omega_{m}\left(z ; w_{0}, \omega_{0}\right)+$ $g\left(z ; w_{m}\left(z ; w_{0}, \omega_{0}\right), \omega_{m}\left(z ; w_{0}, \omega_{0}\right)\right)$.

Consider the Eigen-value of matrix $\Phi(z)$ does not exceed one, while 
$\Phi(z)=\left[\begin{array}{ll}\Phi_{1}(z) & \Phi_{2}(z) \\ \Phi_{3}(z) & \Phi_{4}(z)\end{array}\right]$

Where, $\quad \Phi_{1}(z)=z R_{1}\left(\left\|B_{1}(z)\right\|+\Gamma_{1}\right), \quad \Phi_{2}(z)=$ $z R_{1}\left(\left\|A_{2}+B_{2}(z)\right\|+\Gamma_{2}\right), \quad \Phi_{3}(z)=z R_{2}\left(\| C_{1}+\right.$ $\left.D_{1}(z) \|+\Sigma_{1}\right)$ and $\Phi_{4}(z)=z R_{2}\left(\left\|D_{2}(z)\right\|+\Sigma_{2}\right)$

Thus, the maximum value of $\Phi(z)$ has obtained from:

$$
\begin{aligned}
& \max (\Phi(Z))=\frac{\Phi_{1}(Z)+\Phi_{4}(Z)}{2} \\
& +\frac{\sqrt{\left(\Phi_{1}(Z)+\Phi_{4}(Z)\right)^{2}-4\left(\Phi_{1}(Z) \Phi_{4}(Z)-\Phi_{2}(Z) \Phi_{3}(Z)\right)}}{2}<1
\end{aligned}
$$

wherever,

$$
\left.\begin{array}{c}
\varphi_{1}(Z)=\left\|B_{1}(Z)\right\|+\Gamma_{1} \\
\varphi_{2}(Z)=\left\|A_{2}+B_{2}(Z)\right\|+\Gamma_{2} \\
\varphi_{3}(Z)=\left\|C_{1}+D_{1}(Z)\right\|+\Sigma_{1} \\
\varphi_{4}(Z)=\left\|D_{2}(Z)\right\|+\Sigma_{2}
\end{array}\right\}
$$

Definition 1.1. [11] If $w(z)$ has a derivative at each point of region $\Omega$ and is single-valued, it is said to be analytic in a region $\Omega$ of the complex plane.

Definition 1.2. [2] Let the complex vector functions $w\left(0, w_{0}, \omega_{0}\right)$ and $\omega\left(0, w_{0}, \omega_{0}\right)$ are defined and analytic in $\mathbb{C} \times \mathbb{C} \rightarrow \mathbb{C}$. Then the following properties, measures the Euclidean distance and defined the metric space:

1. $\|w\| \geq 0$ and, $\|w\|=0$ if $w=0$.

2. $\|w \cdot \omega\|=\|w\| \cdot\|\omega\|$.

3. $\|w+\omega\|=\|w\|+\|\omega\|$.

Definition 1.3. [10] Let the complex vector function $w$ is defined and analytic in $\mathbb{C}$. Then the set $B_{\rho}(c)=$ $\{w \in \mathbb{C}:\|w-c\| \leq \rho\}, \quad \rho>0$ is called closed disc about c.

Definition 1.4. [10] A function $f: \Omega \rightarrow \mathbb{C}$ is called holomorphic in the domain $\Omega$ if $f$ is complex differentiable at every point of $\Omega$. We say $f$ is holomorphic at $c \in \Omega$ if there is an open neighborhood $U$ of $c$ lying in $\Omega$ such that the restriction $f \mid U$ of $f$ to $U$ is holomorphic in $U$.

Lemma 1.1. [7] Let the complex function $w: \mathbb{C} \rightarrow \mathbb{C}$ be an analytic vector function on a finite interval $[-\alpha, \alpha]$, then there is some $M$ such that $\|w(z)\| \leq$ $M$. Also if we have $w: \mathbb{C}^{2} \rightarrow \mathbb{C}^{2}$ is an analytic vector function on a finite rectangle, $[-\alpha, \alpha] \times[-\beta, \beta]$, then there is an $M$ such that $\|w(z, \omega)\| \leq M$.
Lemma 1.2. Suppose that we define the holomorphic complex-valued vector functions $f\left(z ; w\left(z ; w_{0}, \omega_{0}\right), \omega\left(z ; w_{0}, \omega_{0}\right)\right) \quad$ and $g\left(z ; w\left(z ; w_{0}, \omega_{0}\right), \omega\left(z ; w_{0}, \omega_{0}\right)\right)$ on $[0, Z]$. Then the equations number (3) and (4) are solutions under the complex Integrodifferential equations of system (1).

Proof. Let $w\left(z ; w_{0}, \omega_{0}\right)=\psi\left(z ; w_{0}, \omega_{0}\right) e^{A_{1} z}$ and, $\omega\left(z ; w_{0}, \omega_{0}\right)=\chi\left(z ; w_{0}, \omega_{0}\right) e^{C_{2} z}$. Then from the complex Integrodifferential equation $d w\left(z ; w_{0}, \omega_{0}\right) / d z$ in system (1) we get the form

$$
\begin{aligned}
\psi\left(z ; w_{0}, \omega_{0}\right) A_{1} e^{A_{1} z}+\frac{d \psi\left(z ; w_{0}, \omega_{0}\right)}{d z} e^{A_{1} z} \\
=\left(A_{1}+B_{1}(z)\right) \psi\left(z ; w_{0}, \omega_{0}\right) e^{A_{1} z} \\
+\left(A_{2}+B_{2}(z)\right) \chi\left(z ; w_{0}, \omega_{0}\right) e^{C_{2} z} \\
+f\left(z ; w\left(z ; w_{0}, \omega_{0}\right), \omega\left(z ; w_{0}, \omega_{0}\right)\right) .
\end{aligned}
$$

Simplify the equality to obtain

$$
\begin{aligned}
& \frac{d \psi\left(z ; w_{0}, \omega_{0}\right)}{d z} \\
& =B_{1}(z) \psi\left(z ; w_{0}, \omega_{0}\right) \\
& +\left(A_{2}+B_{2}(z)\right) \chi\left(z ; w_{0}, \omega_{0}\right) e^{C_{2} z} e^{-A_{1} z} \\
& +e^{-A_{1} z} f\left(z ; w\left(z ; w_{0}, \omega_{0}\right), \omega\left(z ; w_{0}, \omega_{0}\right)\right) .
\end{aligned}
$$

Take the integral of both sides from 0 to $z$ and put in, $\quad w\left(z ; w_{0}, \omega_{0}\right)=\psi\left(z ; w_{0}, \omega_{0}\right) e^{A_{1} z} \quad$ where $w\left(z ; w_{0}, \omega_{0}\right)=w_{0}$, to obtain

$$
\begin{aligned}
& w\left(z ; w_{0}, \omega_{0}\right)=w_{0} e^{A_{1} z} \\
& \quad+\int_{0}^{z} e^{A_{1}(z-s)}\left(B_{1}(s) w\left(s, w_{0}, \omega_{0}\right)\right. \\
& \quad+\left(A_{2}+B_{2}(s)\right) \omega\left(z ; w_{0}, \omega_{0}\right) \\
& \left.\quad+f\left(s, w\left(s, w_{0}, \omega_{0}\right), \omega\left(s, w_{0}, \omega_{0}\right)\right)\right) d s .
\end{aligned}
$$

Thus, we confirm the validity of equation number (3), also the equation number (5) by same steps.

Lemma 1.3. Suppose that the complex-valued vector functions $f\left(z ; w\left(z ; w_{0}, \omega_{0}\right), \omega\left(z ; w_{0}, \omega_{0}\right)\right)$ and $g\left(z ; w\left(z ; w_{0}, \omega_{0}\right), \omega\left(z ; w_{0}, \omega_{0}\right)\right)$ are defined on $[0, Z]$. Then they exist and holomorphic over the vector function 
$\left[\begin{array}{l}\left\|E_{1}\left(z ; w_{0}, \omega_{0}\right)\right\| \\ \left\|E_{2}\left(z ; w_{0}, \omega_{0}\right)\right\|\end{array}\right]<\left[\begin{array}{l}\beta_{w} \\ \beta_{\omega}\end{array}\right]$

Whenever, $E_{1}\left(z ; w_{0}, \omega_{0}\right)=\int_{0}^{z} e^{A_{1}(z-s)} \zeta(s) d s$ and, $E_{2}\left(z ; w_{0}, \omega_{0}\right)=\int_{0}^{z} e^{C_{2}(z-s)} \xi(s) d s$.

Proof. From the assumptions (6) and, (10) where, $\|\zeta(z)\| \leq \vartheta_{1}(z),\|\xi(z)\| \leq \vartheta_{2}(z),\left\|e^{A_{1}(z-s)}\right\|=\mathrm{R}_{1}$ and $\left\|e^{C_{2}(z-s)}\right\|=R_{2}$, we have

$\left\|E_{1}\left(z ; w_{0}, \omega_{0}\right)\right\|=\left\|\int_{0}^{z} e^{A_{1}(z-s)} \zeta(s) d s\right\| \leq$ $\int_{0}^{z} e^{\left\|A_{1}(z-s)\right\|}\|\zeta(z)\| d s \leq z R_{1} \vartheta_{1}(z)<\beta_{w}$, and

$\left\|E_{2}\left(z ; w_{0}, \omega_{0}\right)\right\|=\left\|\int_{0}^{z} e^{C_{2}(z-s)} \xi(s) d s\right\| \leq$ $\int_{0}^{z} e^{\left\|C_{2}(z-s)\right\|}\|\xi(z)\| d s \leq z R_{2} \vartheta_{2}(z)<\beta_{\omega}$.

Thence, we have achieved the equation (16).

\section{Existence of solutions of Integrodifferential equations of (1).}

The following theorem clarifies the existence of solutions of system (1).

Theorem 2.1. Suppose the complex-valued vector functions $f\left(z ; w\left(z ; w_{0}, \omega_{0}\right), \omega\left(z ; w_{0}, \omega_{0}\right)\right)$ and $g\left(z ; w\left(z ; w_{0}, \omega_{0}\right), \omega\left(z ; w_{0}, \omega_{0}\right)\right)$ are defined on $(2)$ and satisfy the inequalities (5)-(10) and conditions (13)-(15). Then there exists, sequences of vector functions (11) and (12), that converge uniformly to the limit vector functions $w\left(z ; w_{0}, \omega_{0}\right)$ and $\omega\left(z ; w_{0}, \omega_{0}\right)$ as $m \rightarrow \infty$.

Proof. Firstly, we define the approximate sequences, $w_{m}\left(z ; w_{0}, \omega_{0}\right)$ and $\omega_{m}\left(z ; w_{0}, \omega_{0}\right)$ from the equations (11) and (12) where, $m>0$. Thus by reiterating and from them we have

$\int_{0}^{z}\left\|e^{A_{1}(z-s)}\right\|\left\|\zeta_{m-1}(s)\right\| d s=z R_{1} \vartheta_{1}(z)$

and,

$\int_{0}^{Z}\left\|e^{C_{2}(z-s)}\right\|\left\|\xi_{m-1}(s)\right\| d s=z R_{2} \vartheta_{2}(z)$.

Therefore we obtain that the sequences exist and holomorphic functions in domains $\Omega_{0}$ and, $\Omega_{1}$, then $\left\|w_{m}\left(z ; w_{0}, \omega_{0}\right)-w_{0}\right\|<\beta_{w}$ and, $\left\|\omega_{m}\left(z ; w_{0}, \omega_{0}\right)-\omega_{0}\right\|<\beta_{\omega}$.

Let we achieved for $m<l$, then this implies that $w_{l-1}\left(z ; w_{0}, \omega_{0}\right)$ and $\omega_{l-1}\left(z ; w_{0}, \omega_{0}\right)$ exist and holomorphic in mention domains and satisfy $\left\|w_{l-1}\left(z ; w_{0}, \omega_{0}\right)-w_{0}\right\|<\beta_{w}$ $\left\|\omega_{l-1}\left(z ; w_{0}, \omega_{0}\right)-\omega_{0}\right\|<\beta_{\omega}$. approximate sequences (11) and (12) and for all $l>m$, the results $\left\|w_{l}\left(z ; w_{0}, \omega_{0}\right)-w_{0}\right\|<\beta_{w}$ and, $\left\|\omega_{l}\left(z ; w_{0}, \omega_{0}\right)-\omega_{0}\right\|<\beta_{\omega}$ hold too, because from the composite functions within $\zeta_{l-1}(t)$ and $\xi_{l-1}(t)$ the integral of holomorphic functions are also holomorphic as follows:

$\left\|w_{l}\left(z ; w_{0}, \omega_{0}\right)-w_{0}\right\| \leq\left\|w_{0}\right\|\left\|e^{A_{1} z}-I\right\|+$ $\int_{0}^{z}\left\|e^{A_{1}(z-s)}\right\|\left\|\zeta_{l-1}(s)\right\| d s=Q_{1}(z)+z R_{1} \vartheta_{1}(z)$,

$\left\|\omega_{l}\left(z ; w_{0}, \omega_{0}\right)-\omega_{0}\right\| \leq\left\|\omega_{0}\right\|\left\|e^{C_{2} z}-I\right\|+$ $\int_{0}^{z}\left\|e^{C_{2}(z-s)}\right\|\left\|\xi_{l-1}(s)\right\| d s=Q_{2}(z)+R_{2} \vartheta_{2}(z) z$.

Therefore, $w_{l}\left(z ; w_{0}, \omega_{0}\right)$ and $\omega_{l}\left(z ; w_{0}, \omega_{0}\right)$ are exist and holomorphic in $\Omega_{0}$ and, $\Omega_{1}$.

Secondly, we have to prove that $\left\{w_{m}\left(z ; w_{0}, \omega_{0}\right)\right\}_{m=0}^{\infty} \quad$ and $\quad\left\{\omega_{m}\left(z ; w_{0}, \omega_{0}\right)\right\}_{m=0}^{\infty}$ converge uniformly on domain (2). Thus from (11) and (12) where $m=0$

$\left\|w_{1}\left(z ; w_{0}, \omega_{0}\right)-w_{0}\right\| \leq\left\|w_{0}\right\|\left\|e^{A_{1} z}-I\right\|+$ $z R_{1} \vartheta_{1}(z)$,

$\left\|\omega_{1}\left(z ; w_{0}, \omega_{0}\right)-\omega_{0}\right\| \leq\left\|\omega_{0}\right\|\left\|e^{C_{2} z}-I\right\|+$ $z R_{2} \vartheta_{2}(z)$.

And so on,

$\left\|w_{2}\left(z ; w_{0}, \omega_{0}\right)-w_{1}\left(z ; w_{0}, \omega_{0}\right)\right\| \leq z R_{1} \| \zeta_{1}(s)-$ $\zeta_{0} \|$,

$\left\|\omega_{2}\left(z ; w_{0}, \omega_{0}\right)-\omega_{1}\left(z ; w_{0}, \omega_{0}\right)\right\| \leq z R_{2} \| \xi_{1}(s)-$ $\xi_{0} \|$,

whereas, from Lipschitz conditions (7) and (8)

$\left\|\zeta_{1}(z)-\zeta_{0}\right\| \leq\left(\left\|B_{1}(z)\right\|+\Gamma_{1}\right) \| w_{1}\left(z ; w_{0}, \omega_{0}\right)-$ $w_{0}\left\|+\left(\left\|A_{2}+B_{2}(z)\right\|+\Gamma_{2}\right)\right\| \omega_{1}\left(z ; w_{0}, \omega_{0}\right)-\omega_{0} \|$, $\left\|\xi_{1}(z)-\xi_{0}\right\| \leq$

$\left(\left\|C_{1}+D_{1}(z)\right\|+\Sigma_{1}\right)\left\|w_{1}\left(z, w_{0}, \omega_{0}\right)-w_{0}\right\|+$ $\left(\left\|D_{2}(z)\right\|+\Sigma_{2}\right)\left\|\omega_{1}\left(z, w_{0}, \omega_{0}\right)-\omega_{0}\right\|$.

Thus from the conditions (13), (14) and (15) we obtain that

$\left\|w_{2}\left(z ; w_{0}, \omega_{0}\right)-w_{1}\left(z ; w_{0}, \omega_{0}\right)\right\| \leq$

$\Phi_{1}(z)\left\|w_{1}\left(z ; w_{0}, \omega_{0}\right)-w_{0}\right\|+$

$\Phi_{2}(z)\left\|\omega_{1}\left(z ; w_{0}, \omega_{0}\right)-\omega_{0}\right\|$,

$\left\|\omega_{2}\left(z ; w_{0}, \omega_{0}\right)-\omega_{1}\left(z ; w_{0}, \omega_{0}\right)\right\| \leq$

$\Phi_{3}(z)\left\|w_{1}\left(z ; w_{0}, \omega_{0}\right)-w_{0}\right\|+$

$\Phi_{4}(z)\left\|\omega_{1}\left(z ; w_{0}, \omega_{0}\right)-\omega_{0}\right\|$. 
Consequently for all $m>1$ and by induction, from (13) we obtain the vector form as follows:

$\left[\begin{array}{l}\left\|w_{m+1}\left(z ; w_{0}, \omega_{0}\right)-w_{m}\left(z ; w_{0}, \omega_{0}\right)\right\| \\ \left\|\omega_{m+1}\left(z ; w_{0}, \omega_{0}\right)-\omega_{m}\left(z ; w_{0}, \omega_{0}\right)\right\|\end{array}\right] \leq$

$\left[\Phi_{1}(z) \quad \Phi_{2}(z)\right]\left[\left\|w_{m}\left(z ; w_{0}, \omega_{0}\right)-w_{m-1}\left(z ; w_{0}, \omega_{0}\right)\right\|\right]$

$\left[\Phi_{3}(z) \quad \Phi_{4}(z)\right]\left[\begin{array}{l}\left\|\omega_{m}\left(z ; w_{0}, \omega_{0}\right)-\omega_{m-1}\left(z ; w_{0}, \omega_{0}\right)\right\|\end{array}\right]$

Reiterating the vector inequality for all $m$ with respect to $Z$ (the maximal value of $z$ ) from (13) as:

$\left[\begin{array}{l}\left\|w_{m+1}\left(Z, w_{0}, \omega_{0}\right)-w_{m}\left(Z, w_{0}, \omega_{0}\right)\right\| \\ \left\|\omega_{m+1}\left(Z, w_{0}, \omega_{0}\right)-\omega_{m}\left(Z, w_{0}, \omega_{0}\right)\right\|\end{array}\right] \leq$

$\left[\begin{array}{ll}\Phi_{1}(Z) & \Phi_{2}(Z) \\ \Phi_{3}(Z) & \Phi_{4}(Z)\end{array}\right]^{m}\left[\begin{array}{l}\left\|w_{1}\left(Z, w_{0}, \omega_{0}\right)-w_{o}\right\| \\ \left\|\omega_{1}\left(Z, w_{0}, \omega_{0}\right)-\omega_{o}\right\|\end{array}\right]$.

Thus for all numbers, $k>1$, we have achieved

$\left[\begin{array}{l}\left\|w_{m+k}\left(Z, w_{0}, \omega_{0}\right)-w_{m}\left(Z, w_{0}, \omega_{0}\right)\right\| \\ \left\|\omega_{m+k}\left(Z, w_{0}, \omega_{0}\right)-\omega_{m}\left(Z, w_{0}, \omega_{0}\right)\right\|\end{array}\right] \leq$
$\sum_{i=1}^{k}\left[\begin{array}{l}\left\|w_{m+i}\left(Z, w_{0}, \omega_{0}\right)-w_{m+(i-1)}\left(Z, w_{0}, \omega_{0}\right)\right\| \\ \left\|\omega_{m+i}\left(Z, w_{0}, \omega_{0}\right)-\omega_{m+(i-1)}\left(Z, w_{0}, \omega_{0}\right)\right\|\end{array}\right]$.

Therefore, we obtain the form bellow

$\left[\begin{array}{l}\left\|w_{m+k}\left(Z, w_{0}, \omega_{0}\right)-w_{m}\left(Z, w_{0}, \omega_{0}\right)\right\| \\ \left\|\omega_{m+k}\left(Z, w_{0}, \omega_{0}\right)-\omega_{m}\left(Z, w_{0}, \omega_{0}\right)\right\|\end{array}\right] \leq$

$\left[\begin{array}{ll}\Phi_{1}(Z) & \Phi_{2}(Z) \\ \Phi_{3}(Z) & \Phi_{4}(Z)\end{array}\right]^{2^{k} m+\left(2^{k}-1\right)}\left[\begin{array}{l}\left\|w_{1}\left(Z, w_{0}, \omega_{0}\right)-w_{o}\right\| \\ \left\|\omega_{1}\left(Z, w_{0}, \omega_{0}\right)-\omega_{o}\right\|\end{array}\right]$.

Thus (17) holds for all $k$, and it follows that the series

$$
\begin{aligned}
& {\left[\begin{array}{l}
w\left(Z, w_{0}, \omega_{0}\right) \\
\omega\left(Z, w_{0}, \omega_{0}\right)
\end{array}\right]=\left[\begin{array}{l}
w_{0} \\
\omega_{0}
\end{array}\right]} \\
& +\sum_{m=1}^{\infty}\left[\begin{array}{l}
w_{m}\left(Z, w_{0}, \omega_{0}\right)-w_{m-1}\left(Z, w_{0}, \omega_{0}\right) \\
\omega_{m}\left(Z, w_{0}, \omega_{0}\right)-\omega_{m-1}\left(Z, w_{0}, \omega_{0}\right)
\end{array}\right]
\end{aligned}
$$

Converges uniformly in domain (2) and this implies that $w\left(Z, w_{0}, \omega_{0}\right)$ and $\omega\left(Z, w_{0}, \omega_{0}\right)$ are holomorphic.

Moreover, as a result of condition (14) we received that, $\lim _{m \rightarrow \infty} \Phi^{m}(z)=0$, and from (18) consider the limits, $\quad \lim _{m \rightarrow \infty} w_{m+1}\left(z ; w_{0}, \omega_{0}\right)=$ $w\left(z ; w_{0}, \omega_{0}\right)$ and, $\lim _{m \rightarrow \infty} \omega_{m+1}\left(z ; w_{0}, \omega_{0}\right)=$ $\omega\left(z ; w_{0}, \omega_{0}\right)$.

$\lim _{m \rightarrow \infty} f\left(z ; w_{m}\left(z ; w_{0}, \omega_{0}\right), \omega_{m}\left(z ; w_{0}, \omega_{0}\right)\right)=$ Also, $f\left(z ; w\left(z ; w_{0}, \omega_{0}\right), \omega\left(z ; w_{0}, \omega_{0}\right)\right) \quad$ and, $\lim _{m \rightarrow \infty} g\left(z ; w_{m}\left(z ; w_{0}, \omega_{0}\right), \omega_{m}\left(z ; w_{0}, \omega_{0}\right)\right)=$ $g\left(z ; w\left(z ; w_{0}, \omega_{0}\right), \omega\left(z ; w_{0}, \omega_{0}\right)\right)$. Finally we investigate that $w\left(z ; w_{0}, \omega_{0}\right)$ and $\omega\left(z ; w_{0}, \omega_{0}\right)$ are solutions of complex Integrodifferential equations of system (1).

\section{Uniqueness of solutions of integrodifferential equations of (1).}

The complex Integrodifferential equations of (1) have just one solution.

Theorem 3.1. Under all conditions and assumptions of theorem (2.1), the equations (3) and (4) are unique solutions on domain (9).

Proof. Let the complex vector equations $\widehat{w}\left(z ; w_{0}, \omega_{0}\right)$ and $\widehat{\omega}\left(z ; w_{0}, \omega_{0}\right)$ be another solutions of system (1) which are holomorphic, that is

$\widehat{w}\left(z ; w_{0}, \omega_{0}\right)=w_{0} e^{A_{1} z}+\int_{0}^{z} e^{A_{1}(z-s)} \hat{\zeta}(s) d s$

$\widehat{\omega}\left(z ; w_{0}, \omega_{0}\right)=\omega_{0} e^{C_{2} z}+\int_{0}^{z} e^{C_{2}(z-s)} \hat{\xi}(s) d s$

Where, $\quad \hat{\zeta}(z)=B_{1}(z) \widehat{w}\left(z ; w_{0}, \omega_{0}\right)+\left(A_{2}+\right.$ $\left.B_{2}(z)\right) \widehat{\omega}\left(z ; w_{0}, \omega_{0}\right)+$ $f\left(z ; \widehat{w}\left(z ; w_{0}, \omega_{0}\right), \widehat{\omega}\left(z ; w_{0}, \omega_{0}\right)\right)$.

with, $\widehat{w}\left(0, x_{0}, y_{0}\right)=w_{0}$. And

$\hat{\xi}(z)=$

$\left(C_{1}+D_{1}(z)\right) \widehat{w}\left(z ; w_{0}, \omega_{0}\right)+D_{2}(z) \widehat{\omega}\left(z ; w_{0}, \omega_{0}\right)+$ $g\left(z ; \widehat{w}\left(z ; w_{0}, \omega_{0}\right), \widehat{\omega}\left(z ; w_{0}, \omega_{0}\right)\right)$.

with $\widehat{\omega}\left(0, x_{0}, y_{0}\right)=\omega_{0}$ and, $m=0,1,2, \ldots$.

From the difference of equations (3), (19), (4) and (20) we obtain the norms

$\left\|w\left(z ; w_{0}, \omega_{0}\right)-\widehat{w}\left(z ; w_{0}, \omega_{0}\right)\right\| \leq$ $\int_{0}^{z}\left\|e^{A_{1}(z-s)}\right\|\|\zeta(z)-\hat{\zeta}(z)\| d s$, and

$\left\|\omega\left(z ; w_{0}, \omega_{0}\right)-\widehat{\omega}\left(z ; w_{0}, \omega_{0}\right)\right\| \leq$ $\int_{0}^{z}\left\|e^{C_{2}(z-s)}\right\|\|\xi(z)-\hat{\xi}(z)\| d s$.

Thus from the Lipschitz conditions (7) and (8) we verify that $\|\zeta(z)-\hat{\zeta}(z)\| \leq\left(\left\|B_{1}(z)\right\|+\right.$ $\left.\Gamma_{1}\right)\left\|w\left(z ; w_{0}, \omega_{0}\right)-\widehat{w}\left(z ; w_{0}, \omega_{0}\right)\right\|+\left(\| A_{2}+\right.$ $\left.B_{2}(z) \|+\Gamma_{2}\right)\left\|\omega\left(z ; w_{0}, \omega_{0}\right)-\widehat{\omega}\left(z ; w_{0}, \omega_{0}\right)\right\| \quad$ and $\|\xi(z)-\hat{\xi}(z)\| \leq$

$\left(\left\|C_{1}+D_{1}(t)\right\|+\Sigma_{1}\right) \| w\left(z ; w_{0}, \omega_{0}\right)-$

$\widehat{w}\left(z ; w_{0}, \omega_{0}\right)\left\|+\left(D_{2}(t)+\Sigma_{2}\right)\right\| \omega\left(z ; w_{0}, \omega_{0}\right)-$ $\widehat{\omega}\left(z ; w_{0}, \omega_{0}\right) \|$

Therefore from (13) and (14) we gathering the results in a vector form as: 
$\left[\begin{array}{l}\left\|w\left(z ; w_{0}, \omega_{0}\right)-\widehat{w}\left(z ; w_{0}, \omega_{0}\right)\right\| \\ \left\|\omega\left(z ; w_{0}, \omega_{0}\right)-\widehat{\omega}\left(z ; w_{0}, \omega_{0}\right)\right\|\end{array}\right] \leq$

$\left[\begin{array}{ll}\Phi_{1}(z) & \Phi_{2}(z) \\ \Phi_{3}(z) & \Phi_{4}(z)\end{array}\right]\left[\begin{array}{c}\left\|w\left(z ; w_{0}, \omega_{0}\right)-\widehat{w}\left(z ; w_{0}, \omega_{0}\right)\right\| \\ \left\|\omega\left(z ; w_{0}, \omega_{0}\right)-\widehat{\omega}\left(z ; w_{0}, \omega_{0}\right)\right\|\end{array}\right]$.

And hence from condition (14), the greatest Eigen value of matrix, $\Phi(Z)$ is less than one. So we deduce that, $w\left(z ; w_{0}, \omega_{0}\right)=\widehat{w}\left(z ; w_{0}, \omega_{0}\right)$ and, $\omega\left(z ; w_{0}, \omega_{0}\right)=\widehat{\omega}\left(z ; w_{0}, \omega_{0}\right)$. This implies that the complex integrodifferential equations of system (1) have unique solutions.

\section{Stability of solutions of Integrodifferential equations of (1).}

The following theorem investigates the stability of solution of system (1).

Theorem 4.1. Suppose that the holomorphic vector functions $f\left(z ; w_{0}, \omega_{0}\right)$ and $g\left(z ; w_{0}, \omega_{0}\right)$ satisfy the inequalities and conditions in theorem (2.1). Also $\widehat{w}\left(z ; w_{0}, \omega_{0}\right)$ and $\widehat{\omega}\left(z ; w_{0}, \omega_{0}\right)$ be additional solutions of the (1), then $w\left(z ; w_{0}, \omega_{0}\right)$ and $\omega\left(z ; w_{0}, \omega_{0}\right)$ are stable solutions.

Proof. Let the vector solutions $\widehat{w}\left(z ; w_{0}, \omega_{0}\right)$ and $\widehat{\omega}\left(z ; w_{0}, \omega_{0}\right)$ of the system (1) be specified, then we receive that

$\left\|w\left(z ; w_{0}, \omega_{0}\right)-\widehat{w}\left(z ; w_{0}, \omega_{0}\right)\right\| \leq \| w_{0}-$ $\widehat{w}_{0}\|\| e^{A_{1} z}\left\|+\mathrm{R}_{1} z\right\| \zeta(s)-\hat{\zeta}(s) \|$

$\leq\left\|w_{0}-\widehat{w}_{0}\right\|\left\|e^{A_{1} z}\right\|+\mathrm{R}_{1} z\left(\left\|B_{1}(z)\right\|+\right.$ $\left.\Gamma_{1}\right)\left\|w\left(z ; w_{0}, \omega_{0}\right)-\widehat{w}\left(z ; w_{0}, \omega_{0}\right)\right\|+\mathrm{R}_{1} z\left(\| A_{2}+\right.$ $\left.B_{2}(z) \|+\Gamma_{2}\right)\left\|\omega\left(z ; w_{0}, \omega_{0}\right)-\widehat{\omega}\left(z ; w_{0}, \omega_{0}\right)\right\|$

$\leq\left\|w_{0}-\widehat{w}_{0}\right\|\left\|e^{A_{1} z}\right\|+R_{1} z \varphi_{1}(z) \| w\left(z ; w_{0}, \omega_{0}\right)-$ $\widehat{w}\left(z ; w_{0}, \omega_{0}\right)\left\|+R_{1} z \varphi_{2}(z)\right\| \omega\left(z ; w_{0}, \omega_{0}\right)-$ $\widehat{\omega}\left(z ; w_{0}, \omega_{0}\right) \|$

To achieve the norm (22), same procedures are followed. Thus with same duplications we have

$\left\|\omega\left(z ; w_{0}, \omega_{0}\right)-\widehat{\omega}\left(z ; w_{0}, \omega_{0}\right)\right\| \leq \| \omega_{0}-$ $\widehat{\omega}_{0}\|\| e^{C_{2} z}\left\|+R_{2} z \varphi_{3}(z)\right\| w\left(z ; w_{0}, \omega_{0}\right)-$ $\widehat{w}\left(z ; w_{0}, \omega_{0}\right)\left\|+R_{2} z \varphi_{4}(z)\right\| \omega\left(z ; w_{0}, \omega_{0}\right)-$ $\widehat{\omega}\left(z ; w_{0}, \omega_{0}\right) \|$

Collect above inequalities (21) and (22) in a vector form as follows:-

$$
\begin{aligned}
& {\left[\begin{array}{l}
\left\|w\left(z ; w_{0}, \omega_{0}\right)-\widehat{w}\left(z ; w_{0}, \omega_{0}\right)\right\| \\
\left\|\omega\left(z ; w_{0}, \omega_{0}\right)-\widehat{\omega}\left(z ; w_{0}, \omega_{0}\right)\right\|
\end{array}\right]} \\
& \leq\left[\begin{array}{l}
\left\|w_{0}-\widehat{w}_{0}\right\|\left\|e^{A_{1} z}\right\| \\
\left\|\omega_{0}-\widehat{\omega}_{0}\right\|\left\|e^{C_{2} z}\right\|
\end{array}\right] \\
& +\left[\begin{array}{rr}
\varphi_{1}(z) & \varphi_{2}(z) \\
\varphi_{3}(z) & \varphi_{4}(z)
\end{array}\right]\left[\begin{array}{l}
\left\|w\left(z ; w_{0}, \omega_{0}\right)-\widehat{w}\left(z ; w_{0}, \omega_{0}\right)\right\| \\
\left\|\omega\left(z ; w_{0}, \omega_{0}\right)-\widehat{\omega}\left(z ; w_{0}, \omega_{0}\right)\right\|
\end{array}\right] .
\end{aligned}
$$

Thus

$$
\begin{aligned}
& {\left[\begin{array}{l}
\left\|w\left(z ; w_{0}, \omega_{0}\right)-\widehat{w}\left(z ; w_{0}, \omega_{0}\right)\right\| \\
\left\|\omega\left(z ; w_{0}, \omega_{0}\right)-\widehat{\omega}\left(z ; w_{0}, \omega_{0}\right)\right\|
\end{array}\right] \leq} \\
& {\left[\begin{array}{l}
\left\|w_{0}-\widehat{w}_{0}\right\|\left\|e^{A_{1} z}\right\| \\
\left\|\omega_{0}-\widehat{\omega}_{0}\right\|\left\|e^{C_{2} z}\right\|
\end{array}\right]+} \\
& \varphi_{\gamma}^{m}(z)\left[\begin{array}{l}
\left\|w\left(z ; w_{0}, \omega_{0}\right)-\widehat{w}\left(z ; w_{0}, \omega_{0}\right)\right\| \\
\left\|\omega\left(z ; w_{0}, \omega_{0}\right)-\widehat{\omega}\left(z ; w_{0}, \omega_{0}\right)\right\|
\end{array}\right] .
\end{aligned}
$$

From the definition of stability we have, $\left(\begin{array}{l}\left\|w_{0}-\widehat{w}_{0}\right\| \\ \left\|\omega_{0}-\widehat{\omega}_{0}\right\|\end{array}\right) \leq\left(\begin{array}{l}\delta_{1} \\ \delta_{2}\end{array}\right)$, then

$$
\begin{aligned}
& {\left[\begin{array}{l}
\left\|w\left(z ; w_{0}, \omega_{0}\right)-\widehat{w}\left(z ; w_{0}, \omega_{0}\right)\right\| \\
\left\|\omega\left(z ; w_{0}, \omega_{0}\right)-\widehat{\omega}\left(z ; w_{0}, \omega_{0}\right)\right\|
\end{array}\right]} \\
& \leq\left[\begin{array}{cc}
\left\|e^{A_{1} z}\right\| & 0 \\
0 & \left\|e^{C_{2} z}\right\|
\end{array}\right]\left[\begin{array}{l}
\delta_{1} \\
\delta_{2}
\end{array}\right] \\
& +\varphi_{\gamma}^{m}(z)\left[\begin{array}{l}
\left\|w\left(z ; w_{0}, \omega_{0}\right)-\widehat{w}\left(z ; w_{0}, \omega_{0}\right)\right\| \\
\left\|\omega\left(z ; w_{0}, \omega_{0}\right)-\widehat{\omega}\left(z ; w_{0}, \omega_{0}\right)\right\|
\end{array}\right] .
\end{aligned}
$$

Then, $\quad\left[\begin{array}{l}\left\|w\left(z ; w_{0}, \omega_{0}\right)-\widehat{w}\left(z ; w_{0}, \omega_{0}\right)\right\| \\ \left\|\omega\left(z ; w_{0}, \omega_{0}\right)-\widehat{\omega}\left(z ; w_{0}, \omega_{0}\right)\right\|\end{array}\right] \leq\left[\begin{array}{l}\varepsilon_{1} \\ \varepsilon_{2}\end{array}\right]$, where $\left[\begin{array}{l}\delta_{1} \\ \delta_{2}\end{array}\right]=\left[\begin{array}{l}\varepsilon_{1} /\left\|e^{A_{1} z}\right\| \\ \varepsilon_{2} /\left\|e^{C_{2} z}\right\|\end{array}\right]$.

Thus we conclude that, $w\left(z ; w_{0}, \omega_{0}\right)$ and $\omega\left(z ; w_{0}, \omega_{0}\right)$ are stable solutions of system (1).

\section{Numerical results}

In this section, we presented an example to demonstrate and illustrate the validity and certainty of existence and uniqueness of solutions of complex Integrodifferential system (1) as follows [13]:

Example. Let us consider the non-linear system of complex Integrodifferential equations $(\mathrm{P})$ as: 


$$
\left.\begin{array}{rl}
\frac{d w}{d z}=(2 & +3 i) z \cos (w(z))+i z \omega(z) \\
& +\int_{0}^{z} e^{i(z-s)} \omega(s) d s \\
\frac{d \omega}{d z}=z & w(z)+(1-i) z \sin (\omega(z)) \\
+ & \int_{0}^{z} e^{-i(z-s)} w(s) d s
\end{array}\right\}
$$

with initial conditions, $w_{0}=i$ and, $\omega_{0}=0.5+1.5 i$ consequently.

Solution. Firstly, if $w(z)=u(z)+i v(z)$ and $\omega(z)=\alpha(z)+i \beta(z)$ be complex functions, then from table below we represent the complex-valued functions $w(z)$ and $\omega(z)$ in real and imaginary

\begin{tabular}{|c|c|c|c|}
\hline $\begin{array}{l}\text { Time } \\
(\mathrm{t})\end{array}$ & $\mathrm{z}$ & $w(z)$ & $\omega(z)$ \\
\hline 0 & $\begin{array}{c}0.0000+ \\
0.0000 \mathrm{i}\end{array}$ & $0.0000+1.0000 \mathrm{i}$ & $0.5000+1.5000 \mathrm{i}$ \\
\hline 0.3142 & $\begin{array}{c}0.3142+ \\
0.3142 \mathrm{i}\end{array}$ & $0.1289+1.3602 \mathrm{i}$ & $0.6716+1.6361 \mathrm{i}$ \\
\hline 0.6283 & $\begin{array}{c}0.6283+ \\
0.6283 i\end{array}$ & $1.5574+2.1061 \mathrm{i}$ & $1.4467+1.9242 \mathrm{i}$ \\
\hline 0.9425 & $\begin{array}{c}0.9425+ \\
0.9425 \mathrm{i}\end{array}$ & $2.2088+1.2218 \mathrm{i}$ & $2.5697+1.6499 \mathrm{i}$ \\
\hline 1.2566 & $\begin{array}{c}1.2566+ \\
1.2566 \mathrm{i}\end{array}$ & $2.2402+1.0882 \mathrm{i}$ & $3.3387+1.4841 \mathrm{i}$ \\
\hline 1.5708 & $\begin{array}{c}1.5708+ \\
1.5708 \mathrm{i}\end{array}$ & $2.2533+1.1712 \mathrm{i}$ & $3.7876+1.7657 i$ \\
\hline 1.8850 & $\begin{array}{c}1.8850+ \\
1.8850 \mathrm{i}\end{array}$ & $2.2265+1.2095 \mathrm{i}$ & $3.6191+1.9235 i$ \\
\hline 2.1991 & $\begin{array}{c}2.1991+ \\
2.1991 \mathrm{i}\end{array}$ & $2.2350+1.2308 \mathrm{i}$ & $3.7542+1.7323 i$ \\
\hline 2.5133 & $\begin{array}{c}2.5133+ \\
2.5133 \mathrm{i}\end{array}$ & $2.2103+1.2589 i$ & $3.7958+1.7618 \mathrm{i}$ \\
\hline 2.8274 & $\begin{array}{c}2.8274+ \\
2.8274 \mathrm{i}\end{array}$ & $2.1951+1.2649 \mathrm{i}$ & $3.8563+1.6197 i$ \\
\hline 3.1416 & $\begin{array}{c}3.1416+ \\
3.1416 \mathrm{i}\end{array}$ & $2.1676+1.2647 i$ & $3.8676+1.5686 \mathrm{i}$ \\
\hline 3.4557 & $\begin{array}{c}3.4558+ \\
3.4558 \mathrm{i} \\
\end{array}$ & $2.1479+1.2477 \mathrm{i}$ & $3.9100+1.4315 i$ \\
\hline 3.7699 & $\begin{array}{c}3.7699+ \\
3.7699 \mathrm{i}\end{array}$ & $2.1236+1.2208 \mathrm{i}$ & $3.8792+1.3376 \mathrm{i}$ \\
\hline 4.0841 & $\begin{array}{c}4.0841+ \\
4.0841 \mathrm{i} \\
\end{array}$ & $2.1053+1.1803 i$ & $3.8745+1.2121 \mathrm{i}$ \\
\hline 4.3982 & $\begin{array}{c}4.3982+ \\
4.3982 \mathrm{i} \\
\end{array}$ & $2.0866+1.1305 \mathrm{i}$ & $3.8050+1.1201 \mathrm{i}$ \\
\hline$\vdots$ & $\vdots$ & $\vdots$ & $\vdots$ \\
\hline 9.1106 & $\begin{array}{c}9.1106+ \\
9.1106 \mathrm{i}\end{array}$ & $2.1114+0.9745 i$ & $3.6183+1.3482 \mathrm{i}$ \\
\hline 9.4248 & $\begin{array}{c}9.4248+ \\
9.4248 \mathrm{i} \\
\end{array}$ & $2.1037+0.9848 i$ & $3.6334+1.3175 i$ \\
\hline
\end{tabular}
parts.

Table 1. Numerical data of approximate solutions of $w(z)$ and $\omega(z)$ :

Numerically through data of table 1 we figure the problem geometrically as:

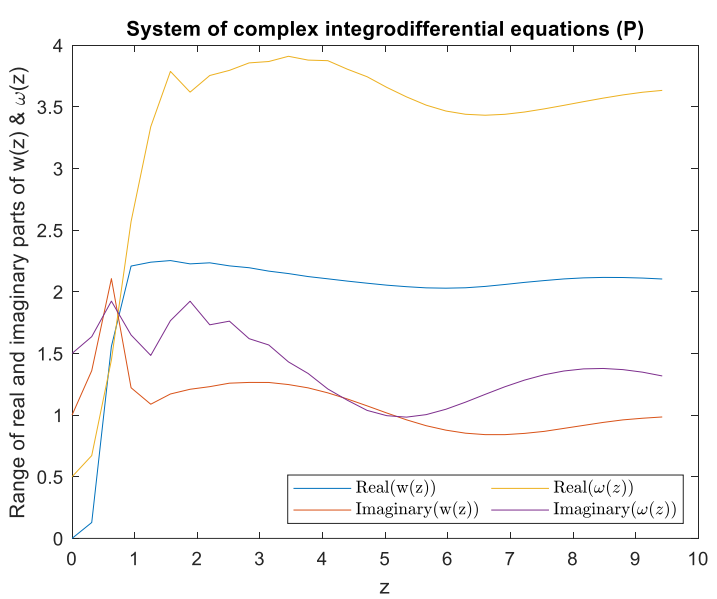

Fig.1. Curves of real and imaginary parts of $w(z)$ and, $\omega(z)$

\section{Conclusion}

System of complex Integrodifferential equations is usually difficult to solve exactly, then it is demanded to obtain the approximate solutions. In this work we have to prove the existence, uniqueness and stability of solutions under the necessary and sufficient assumptions on complex Euclidean $n$ spaces $\left(\mathbb{C}^{n}\right)$ and through an example verifies the validity of them. In addition, through Matlab code we have to solve the system of linear and nonlinear Integrodifferential equations in complex and real analysis, in which some modifications are required in code.

\section{HHHQFHWD}

[1] Agarwal, P., Akbar, M., Nawaz, R., \& Jleli, M. (2021). Solutions of system of Volterra integrodifferential equations using optimal homotopy asymptotic method. Mathematical Methods in the Applied Sciences, 44(3), 2671-2681. doi:https://doi.org/10.1002/mma.6783

[2] Barry Simon. (2015). Advanced Complex Analysis: A Comprehensive Course in Analysis, Part 2B (Vol. 2). Simon. doi:https://doi.org/10.1090/simon/002.2

[3] Butris, R. N., Faris, H. S. (2020). Periodic solutions for nonlinear systems of multiple integro-integral differential equations of $(\mathrm{V} F)$ and $(\mathrm{F} \mathrm{V})$ type with isolated singular kernels. General Letters in Mathematics (GLM ), 9(2), 106- 128. doi:10.31559/glm

[4] Costin, O., Tanveer, S. (2000). Existence and uniqueness for a class of nonlinear higher-order partial differential equations in the complex plane. Communications on Pure and Applied Mathematics, 53(9), 1092-1117. doi:https://doi.org/10.1002/10970312(200009)53:9\%3C 1092::aid-cpa2\%3E3.0.co;2-z 
[5] Erfanian, M., Zeidabadi, H. (2019). Approximate solution of linear Volterra integro-differential equation by using cubic B-spline finite element method in the complex plane. Advances in Difference Equations, 2019(1), 1-12. doi:https://doi.org/10.1186/s13662-0192012-9

[6] Hajighasemi, S., Allahviranloo, T., Khezerloo, M., Khorasany, M., Salahshour, S. (June, 2010). Existence and uniqueness of solutions of fuzzy Volterra integrodifferential equations. International Conference on Information Processing and Management of Uncertainty in Knowledge-Based Systems (pp. 491-500). Berlin, Heidelberg. : Springer. doi:https://doi.org/10.1007/9783-642-14058-7_51

[7] Laine, I. (2008). Complex differential equations. Handbook of Differential Equations: Ordinary Differential Equations, 4, 269-363. doi:https://doi.org/10.1016/S1874-5725(08)80008-9

[8] Needham, D. J., King, A. C. (1994). On meromorphic complex differential equations . Dynamics and Stability of Systems, 9(2), 99-122. doi:https://doi.org/10.1080/02681119408806171

[9] Orlov, V., Kovalchuk, O. (June, 2018). Research of one class of nonlinear differential equations of third order for mathematical modelling the complex structures. IOP Conference Series: Materials Science and Engineering. $365, \quad$ pp. 42-45. IOP. doi:https://doi.org/10.1088/1757-899x/365/4/042045

[10] Remmert, R. (1991). Theory of complex functions (Vol. 122). Springer Science \& Business Media. .

[11] Spiegel, M. R., Lipschutz, S., Schiller, J. J., Spellman, D. (2015). Complex variables: With an introduction to conformal mapping and its applications . McGraw-Hill.
[12] Tari, A., Shahmorad, S. (2011). Differential transform method for the system of two-dimensional nonlinear Volterra integro-differential equations. Computers \& Mathematics with Applications , 61(9), 2621-2629.

doi:https://doi.org/10.1016/j.camwa.2011.03.007

[13] Toutounian, F., Tohidi, E., Shateyi, S. (2013). A collocation method based on the Bernoulli operational matrix for solving high-order linear complex differential equations in a rectangular domain. Abstract and Applied Analysis. doi:https://doi.org/10.1155/2013/823098

[14] Vanualailai, J., Nakagiri, S. I. (2003). Stability of a system of Volterra integro-differential equations. Journal of mathematical analysis and applications, 281(2), 602619. doi:https://doi.org/10.1016/s0022-247x(03)001719

Raad Noori Butirs was the supervisor of this article and was responsible for organizing it.

Hewa Selman Faris has implemented the code and Algorithm of the Matlab function for solving the system of complex integrodifferential equations $(\mathrm{P})$ and write the article.

\section{Creative Commons Attribution License 4.0 (Attribution 4.0 International, CC BY 4.0)}

This article is published under the terms of the Creative Commons Attribution License 4.0 https://creativecommons.org/licenses/by/4.0/deed.en US 